\title{
COVID-19 EN AMÉRICA LATINA: MÁS ALLÁ DE LOS DATOS EPIDEMIOLÓGICOS
}

\section{COVID-19 in Latin America: Beyond Epidemiological Data}

\author{
Óscar ARTEAGA HERRERA; Alejandra FUENTES GARCÍA \\ Escuela de Salud Pública, Facultad de Medicina, Universidad de Chile (Chile). \\ Correo electrónico: oarteaga@uchile.cl
}

Fecha de recepción: 2 de octubre de 2020

Fecha de aceptación: 7 de octubre de 2020

Fecha de publicación: 29 de enero de 2021

\begin{abstract}
Resumen
En este artículo analizamos el escenario sanitario de la epidemia de COVID-19 en América Latina y profundizamos en algunos aspectos relevantes del escenario político, económico y social que caracteriza a la región en los momentos en que está transcurriendo esta pandemia. En América Latina se concentra más del $50 \%$ de casos de COVID-19 registrados en el mundo, esta región tiene los países con las mayores tasas de letalidad y mortalidad por el virus. La pandemia ha transcurrido en un escenario de situaciones políticas extremadamente complejas y precarias, con situaciones económicas críticas y brutales desigualdades de género en prácticamente todos los países de la región. La pandemia en cuanto evento profundizador de las desigualdades sociales y develador de su crudeza y acción, incrementa mayor visibilidad de estas desigualdades que pueden generar demanda social de cambio y reconocimiento de la consagración de la salud como un derecho humano. La importancia de la salud pública y la necesidad de fortalecer los sistemas públicos de salud se ha hecho más evidente y necesaria que nunca.

Palabras clave: COVID-19; América latina; desigualdades; morbilidad; mortalidad.
\end{abstract}

\section{Abstract}

In this article we analyze the health situation related to the COVID-19 epidemic in Latin America and deepen into relevant aspects of the political, economic and social scenario that 


\section{COVID-19 EN AMÉRICA LATINA: MÁS ALLÁ DE LOS DATOS EPIDEMIOLÓGICOS \\ ÓSCAR ARTEAGA HERRERA; ALEJANDRA FUENTES GARCÍA}

characterizes the region at the time this pandemic is unfolding. More than $50 \%$ of COVID-19 cases registered in the world are concentrated in Latin America; this region has the countries with the highest fatality and mortality rates from the virus. The pandemic has taken place in a scenario of extremely complex and precarious political situations, with critical economic situations and brutal gender inequalities in practically all the countries of the region. The pandemic as an event that deepens social inequalities and reveals its harshness and action, increases the visibility of these inequalities that can generate social demand for change and recognition of the consecration of health as a human right. The importance of public health and the need to strengthen public health care systems has become more evident and necessary than ever.

Keywords: COVID-19; Latin America; inequalities; morbidity; mortality.

\section{INTRODUCCIÓN}

En la serie de televisión de Nexflix "La catedral del Mar", en una escena ambientada en el año 1350, un vocero del gobierno municipal de la ciudad de Barcelona lee un comunicado oficial que informa: "En nombre del Consejo de Ciento, se hace saber que en la pasada semana, los muertos por pestilencia en Barcelona solo ascendieron a 32. Nueve personas han sido curadas y sus casas han sido declaradas libres de pestilencia"1.

La escena indicada, versión medieval de los comunicados de la autoridad sanitaria en cualquier país actual informando por televisión la situación de COVID-19, se refiere a la epidemia de Peste Negra, producida por Yersinia pestis, siendo las pulgas los vectores en cuyo intestino se multiplica la bacteria y que a mediados del siglo XIV habría provocado la muerte de alrededor de 50 millones de personas en Europa ${ }^{2}$. Los más afectados por la epidemia fueron personas cuya cotidianeidad de vida implicaba mayor contacto con ratas, que portaban a las pulgas ${ }^{3}$, es decir las personas viviendo en pobreza, cuyas condiciones de salud podrían, además, haber estado afectadas por situaciones de repetidas hambrunas en las décadas anteriores a $1350^{4}$. El patrón no es distinto en la pandemia de gripe H1N1 de 1918 (gripe española), respecto de la cual se ha demostrado que las personas con tuberculosis, una enfermedad que es un indicador de pobreza, tuvieron mayor probabilidad de morir de influenza ${ }^{5}$. Este mismo patrón de mayor y más severo impacto en personas y poblaciones social y económicamente más vulnerables se repite con COVID-19. Esta pandemia, cuyo origen se inició en Wuhan el 17 de noviembre de 2019, causado por el SARS-CoV- $2^{6}$, en 11 meses de ocurrencia ha acumulado 30.055 .710 casos con 943.433 personas fallecidas de la enfermedad ${ }^{7}$. Más de la mitad de esas muertes se concentra en América Latina, una de las regiones del mundo con mayores desigualdades y pobreza.

Más allá del impacto sanitario, las consecuencias sociales por la pandemia del COVID-19 han sido dramáticas. El Banco Mundial ha estimado que el número de personas que viven por debajo de las líneas internacionales de pobreza para los países de ingresos bajos y medio-altos (USD 3,20/día y USD 5,50/día; corregido por paridad de poder de compra) podrían incrementarse en 176 millones para el nivel USD 3,20 y 177 millones para el nivel USD 5,501. Entre estos nuevos pobres, una gran proporción se concentraría en países que ya enfrentaban elevadas cifras de pobreza desde antes de la pandemia ${ }^{8}$ como es el caso de América Latina. Las Naciones Unidas han estimado una contracción del Producto Interno Bruto (PIB) de 9,1\% en 2020 en la región como consecuencia de COVID-19, lo que incrementaría el número de pobres en 45 millones para llegar a alcanzar los 230 millones de personas viviendo en condiciones de pobreza; las personas en extrema pobreza aumentarían en 28 millones para llegar a totalizar 96 millones $^{9}$. Lo anterior, sumado a las desigualdades sociales 


\section{COVID-19 EN AMÉRICA LATINA: MÁS ALLÁ DE LOS DATOS EPIDEMIOLÓGICOS \\ ÓSCAR ARTEAGA HERRERA; ALEJANDRA FUENTES GARCÍA}

y las crisis políticas que caracterizan a la región, constituye una amenaza cierta que podría potenciar una exacerbación de las crisis sociales.

Como toda epidemia vivida por la humanidad, el COVID-19 es un evento histórico, que, en palabras de Charles Rosenberg, "como fenómeno social tiene una forma dramatúrgica", pues las "epidemias comienzan en un momento en el tiempo, continúan en un escenario limitado en espacio y duración, siguen una línea argumental de tensión creciente y reveladora, se mueven hacia una crisis de carácter individual y colectivo, luego derivan hacia el cierre"10.

Tomando la metáfora que nos ofrece este historiador de la Medicina, es que en este artículo buscamos describir y analizar cómo está transcurriendo la pandemia COVID-19 en América Latina y sus características e impactos. Para ello, desde la mirada interdisciplinar de la salud pública, analizamos primero la dimensión sanitaria de la epidemia, considerando aspectos epidemiológicos y de los sistemas sanitarios, para luego profundizar en el contexto social, económico y político que establece el escenario donde transcurre esta epidemia. Al final del artículo planteamos posibles lecciones que, desde nuestra perspectiva, puede dejar la pandemia en cuanto evento profundizador de las desigualdades sociales y develador de su crudeza y acción.

\section{ANTECEDENTES SANITARIOS DE COVID-19 EN} AMÉRICA LATINA

En el total global de casos de COVID-19, a mediados de septiembre, la región de las Américas acumulaba $51 \%$ de todos los casos confirmados en el mundo y $55 \%$ del total de las defunciones, participación bastante más alta que Asia Sudoriental (19\% del total de casos y $10 \%$ del total de defunciones) y Europa ( $17 \%$ del total de casos y $25 \%$ de las defunciones totales ${ }^{11}$.

A la fecha del 15 de septiembre, en los 54 países y territorios de la Región de las Américas se habían notificado 14.903.891 casos de
COVID-19 y 513.246 defunciones, habiéndose incrementado en $21 \%$ los casos y en $17 \%$ las defunciones en las últimas tres semanas previas a dicha fecha ${ }^{11}$. Si bien este incremento de casos y muertes se ha observado en todas las subregiones de las Américas, el comportamiento en cada una ellas ha sido diferente. En efecto, el mayor aumento entre el 26 de agosto y 15 de septiembre ocurrió en América Central con $28 \%$ de aumento en casos y $22 \%$ de aumento de defunciones, mientras que el más bajo estuvo en América del Norte con $16 \%$ de incremento en casos y $12 \%$ en defunciones. América del Sur e Islas del Caribe y del Océano Atlántico registraron $26 \%$ y $24 \%$ de aumento de casos y $23 \%$ y $34 \%$ de aumento de las defunciones, respectivamente $^{11}$ (figuras 1 y 2 ).

Entre los 10 países con mayor número de casos acumulados de COVID-19 en el mundo, cinco corresponden a países latinoamericanos: Brasil, Colombia, Perú, México y Argentina ${ }^{12}$. Al considerar las tasas de mortalidad por COVID19 , es decir número de personas fallecidas por cada 100 mil habitantes, nuevamente cinco países latinoamericanos se ubican entre los 10 con mayor mortalidad: Perú, con 100,48 /100 mil es el segundo país con la mayor mortalidad en el mundo; Bolivia $(69,21 / 100$ mil; cuarto lugar

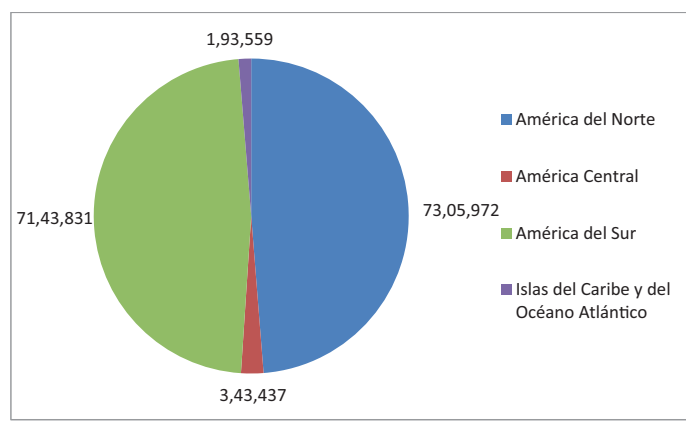

Figura 1. Distribución de casos de COVID-19. Subregiones de las Américas. 15 septiembre 2020

Fuente: Elaboración propia en base a información de OPS/OMS. Actualización Epidemiológica Enfermedad por coronavirus (COVID-19) 


\section{COVID-19 EN AMÉRICA LATINA: MÁS ALLÁ DE LOS DATOS EPIDEMIOLÓGICOS \\ ÓSCAR ARTEAGA HERRERA; ALEJANDRA FUENTES GARCÍA}

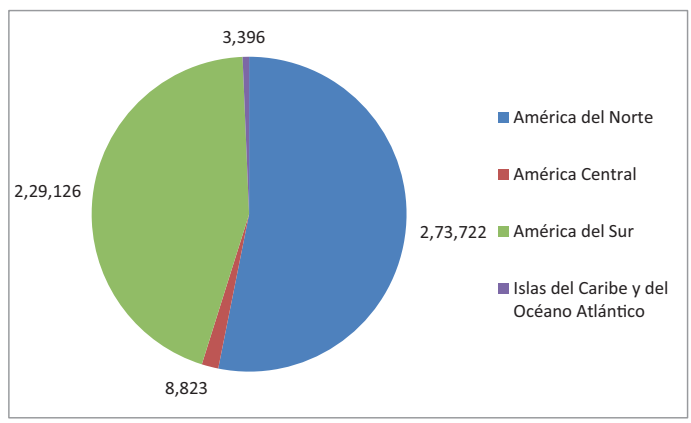

Figura 2. Distribución de fallecidos de COVID-19. Subregiones de las Américas. 15 septiembre 2020

Fuente: Elaboración propia en base a información de OPS/OMS. Actualización Epidemiológica Enfermedad por coronavirus (COVID-19).

en el mundo); Brasil (sexto lugar con 67,67/100 mil); Chile (séptimo lugar con $67,49 / 100$ mil) y; Ecuador (noveno lugar con 66,02/100 mil) ${ }^{12}$. El análisis de la letalidad de la enfermedad, es decir el número de personas que fallecen entre quienes son reportados con COVID-19 vuelve a ubicar a cinco países latinoamericanos entre los primeros 10 países con mayor letalidad: México, que con $10,5 \%$ es el país con mayor letalidad en el mundo; Bolivia, que es el segundo con 5,9\%; Perú (sexto lugar con 4,0\%); Colombia (noveno con 3,1 \%) y; Brasil (décimo con 3,0 \%) ${ }^{12}$ (figura 3).

Una de las características de América Latina es la multietnicidad, representada por diversos pueblos originarios y afro descendientes en los países de la región. Al respecto, la mayor cantidad de casos confirmados y defunciones por COVID19 informados en pueblos indígenas se concentra en Brasil (25.814 casos), Colombia (15.537 casos) y Perú ( 14.550 casos) ${ }^{11}$.

Al igual que en las grandes pandemias que han afectado a la humanidad, como las ya mencionadas peste negra y gripe española, COVID-19 también ha golpeado con mayor fuerza en poblaciones socialmente vulnerables. Una característica de la actual pandemia es que la población más afectada corresponde a adultos mayores. En

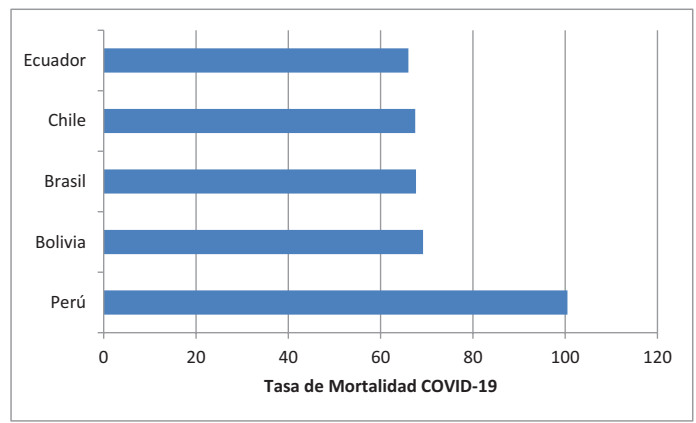

Figura 3. Países con mayor mortalidad por COVID-19 en América Latina. Tasa por 100 mil habitantes.

Fuente: Elaboración propia en base a información de Johns Hospkins University. Coronavirus Resource Center. 2020

Inglaterra, con una letalidad general de la enfermedad de $0,9 \%$, es decir todas las personas que fallecen entre quienes contraen la enfermedad, la letalidad en menores de 44 años es cercana a cero, mientras que esta se incrementa a $3,1 \%$ en el grupo de 65-74 años y a 11,6 \% en personas mayores a los 74 años $^{13}$. En España, los resultados son similares, con una letalidad general de $0,8 \%$, la letalidad en menores de 50 años también es cercana a cero, incrementándose hasta 11,6\% en hombres de 80 y más años ${ }^{13}$. Al comparar entre hombres y mujeres, los hombres tienen mayor probabilidad de morir de COVID-19 que las mujeres, diferencia que se incrementa con la edad ${ }^{13}$.

Desde una perspectiva de salud pública, siempre importa conocer los factores que pueden determinar las enfermedades, especialmente las condiciones sociales en que las personas viven y trabajan, y que conocemos como determinantes sociales de la salud ${ }^{14}$. Al considerar esta perspectiva, encontramos que, si bien los adultos mayores son una población de mayor riesgo, como se ha señalado, el riesgo no es igual en todos los grupos de adultos mayores, sino que el riesgo es mayor en poblaciones más pobres. En el caso de Chile, en la capital Santiago, ciudad territorialmente segregada de acuerdo a nivel socio-económico, 


\section{COVID-19 EN AMÉRICA LATINA: MÁS ALLÁ DE LOS DATOS EPIDEMIOLÓGICOS \\ ÓSCAR ARTEAGA HERRERA; ALEJANDRA FUENTES GARCÍA}

en aquellas comunas de más altos ingresos (ej. Vitacura), el porcentaje de población mayor de 70 años que ha muerto por COVID-19 es $0,5 \%$, mientras que en comunas pobres (ej. Cerro Navia, San Ramón, Puente Alto, Huechuraba, entre otras), esta tasa de mortalidad es al menos tres veces más alta ${ }^{15}$.

La experiencia de enfrentar el desafío del COVID-19 en los sistemas sanitarios latinoamericanos, caracterizados por su segmentación y fragmentación, inequidades en el acceso e ineficiencias en la atención ${ }^{16}$, ha incrementado la necesidad de fortalecer sistemas de salud integrados y coordinados. Sobre esta base, el fortalecimiento de redes integradas de servicios de salud ${ }^{17}$, puede permitir mejorar la equidad de acceso, a la vez que fortalecer la continuidad de la atención sanitaria. Dentro de este enfoque, un aspecto crucial es el fortalecimiento de la atención primaria de salud. La desarticulación de la atención primaria respecto de los otros niveles de atención, o de los niveles de decisión de salud pública, producto de reformas ocurridas en décadas pasadas en los países de América Latina, ha sido un obstáculo para una respuesta sanitaria integral a la epidemia. Esto ha sido especialmente notorio en las dificultades que han existido para la trazabilidad de casos y contactos de COVID-19. Un nivel primario de atención fuerte y resolutivo, trabajando coordinadamente con poblaciones de las que realmente se hace cargo, podría haber permitido un mejor control de la epidemia.

En la siguiente sección de este artículo, se profundiza en las dimensiones económica, social y política relacionadas con la pandemia de COVID-19 en América Latina.

\section{EL ESCENARIO POLÍTICO, ECONÓMICO Y SOCIAL DE LA PANDEMIA DE COVID-19 EN AMÉRICA LATINA}

La irrupción de la pandemia sorprende a muchos países latinoamericanos en medio de situaciones políticas extremadamente complejas y precarias, con gobiernos con muy bajos niveles de apoyo ciudadano, desconfianza de la ciudadanía en las capacidades de los gobiernos e inequidades e injusticias sociales. En el caso de Chile, un país al que se le reconoce una democracia estable, después del estallido social ocurrido en octubre de 2019 contra las desigualdades e injusticias sociales acumuladas a lo largo de los años, los niveles de aprobación del gobierno cayeron a $6 \%$ y la desaprobación alcanzó un $82 \%^{18}$, la evaluación de gobierno más baja desde la recuperación democrática en 1990. En Perú, el presidente Pedro Pablo Kuczynki, que había sido elegido en 2016, debió renunciar en marzo de 2018, siendo reemplazado por Martín Alberto Vizcarra, quien era primer vice-presidente. En Bolivia, el presidente Evo Morales, elegido en 2006 por primera vez, debió renunciar en 2019 asumiendo la senadora Jeanine Añez como Presidenta de la República encabezando un gobierno de transición. En Brasil, el gobierno de Jair Bolsonaro ha transcurrido en medio de diversas controversias, antes y durante la pandemia. La crítica situación de Venezuela se ha extendido ya por varios años. En términos más amplios, el respaldo ciudadano a la democracia en América Latina ha ido disminuyendo progresivamente desde $66,4 \%$ en 2014 a $48 \%$ en $2018^{19}$. En este contexto de graves problemas de gobernanza de los países latinoamericanos, resulta evidente que la conducción de la respuesta a la crisis sanitaria, social y económica generada por COVID-19 se vea aún más dificultada por la reducida legitimidad de los gobiernos.

El impacto económico y social de COVID19 en América Latina es enorme, lo que viene a agravar una situación que antes de la epidemia ya era crítica. En efecto, la región latinoamericana históricamente arrastra altos niveles de desigualdades, con sistemas de impuestos que son regresivos, restricciones en las balanzas de pago de los países, exportaciones concentradas en productos de baja tecnología, todo lo cual se expresa en recurrentes crisis de la deuda externa, 


\section{COVID-19 EN AMÉRICA LATINA: MÁS ALLÁ DE LOS DATOS EPIDEMIOLÓGICOS \\ ÓSCAR ARTEAGA HERRERA; ALEJANDRA FUENTES GARCÍA}

altos niveles de informalidad en el empleo, bajos niveles de crecimiento económico, así como una importante vulnerabilidad al cambio climático, desastres naturales y pérdida de biodiversidad ${ }^{9}$.

La pandemia de COVID-19 se instala en América Latina en un periodo en el cual las economías de los países ya venían con importantes dificultades. En efecto, el sexenio 2014-2019 había registrado solo un $0,4 \%$ de crecimiento, el más bajo desde 1951; la deuda pública había crecido desde $30 \%$ del PIB entre 2009 y 2011 a más de $45 \%$ en $2019^{9}$. En este contexto, detrás de las estimaciones de caída del PIB de 9,1 \% que la Comisión Económica para América Latina y el Caribe (CEPAL) hace para América Latina derivado del impacto económico de COVID-199 ${ }^{9}$, los factores que estarían determinando esta situación serían una caída de las exportaciones de alrededor de $20 \%$ del PIB, disminución de las remesas a la región, también de una magnitud de alrededor de $20 \%$ del PIB, y una menor demanda en el sector turístico ${ }^{9}$. Este último factor afectaría con mayor fuerza al Caribe, región en la cual flujo de turistas ya se habría reducido en alrededor de $40 \%$ en los primeros cuatro meses del presente año ${ }^{9}$.

Las mujeres latinoamericanas se están viendo especialmente afectadas por las consecuencias sociales y económicas de COVID-19. En relación con el empleo, dado que las mujeres constituyen la mayor proporción de trabajadores en servicios de hotelería y alimentación (60\% de los empleado/as), el desempleo asociado a la crisis del sector turístico las afecta más que a los hombres ${ }^{9}$. En términos más amplios, de acuerdo con información del Banco Interamericano de Desarrollo (BID), a partir de una encuesta desarrollada por este organismo y la Universidad de Cornell, tanto las mujeres solteras como casadas, perdieron su trabajo o cerraron su propio negocio en porcentaje mayor que los hombres de esos mismos grupos ${ }^{20}$. Más aún, la pandemia ha exacerbado las desigualdades en la distribución del trabajo doméstico entre hombres y mujeres, pues dos tercios de las mujeres serían las únicas a cargo de realizar las tareas de limpieza y cocina, lo que contrasta con menos de un cuarto de los hombres que lo hacen. A lo anterior se agrega que $60 \%$ de las mujeres reporta ser quienes han debido absorber la carga adicional del cuidado de los niños, en tanto en el caso de los hombres, este porcentaje solo llega al $14 \%^{20}$.

La violencia de género, incluyendo femicidios, alcanza altas cifras en los países de la región, la que se estima entre $10,3 \%$ y $27,2 \%$ de mujeres que informan haber sufrido violencia sexual alguna vez en su vida, perpetrada principalmente por hombres conocidos por ellas ${ }^{21}$. Las medidas adoptadas por los gobiernos para intentar mitigar el impacto sanitario de la epidemia, como el confinamiento también han contribuido a agravar la situación de violencia de género' ${ }^{9}$. Los factores que, durante el confinamiento, han influido en la situación de mayor vulnerabilidad de las mujeres frente al abuso emocional, físico y sexual incluyen la pérdida del poder de negociación en el hogar asociada a la reducción de ingresos, aumento del nivel de contacto interpersonal en el hogar, estrés propio del aislamiento generado por el confinamiento y angustias asociadas a la inseguridad respecto a la capacidad del sistema de salud de responder cuando se le requiera ${ }^{20}$.

\section{AlgunAS LECCIONES QUE NOS DEJA LA PANDEMIA}

La pandemia de COVID-19 vuelve a evidenciar que se trate de enfermedades crónicas o de enfermedades transmisibles, sea en la Edad Media o en esta época contemporánea, las poblaciones más afectadas son las mismas de siempre, es decir aquellas que viven en condiciones de pobreza y marginación, lo que las deja más expuestas y más vulnerables a contagiarse, enfermar o morir. Estas desigualdades, generadas por condicionantes sociales estructurales tales como los tipos de democracia, economía, y las formas en que se distribuye el poder y los recursos en

Rev. Med. Cine. 2020; 16 (e), 119-127 Ediciones Universidad de Salamanca / @®@® J. Med. Mov., 2020; 16 (e), 119-127 


\section{COVID-19 EN AMÉRICA LATINA: MÁS ALLÁ DE LOS DATOS EPIDEMIOLÓGICOS \\ ÓSCAR ARTEAGA HERRERA; ALEJANDRA FUENTES GARCÍA}

una sociedad, se han agudizado y develado durante la pandemia de COVID-19. La visualización de las desigualdades, es decir que un mayor número de personas esté tomando conciencia de las inequidades que antes no les resultaban evidentes y que se arrastran por tanto tiempo en nuestras sociedades, podría estar abriendo una ventana de oportunidad para generar cambios que permitan actuar sobre estos determinantes estructurales.

En el contexto de crisis social, económica y política agudizado por la pandemia que vive América Latina, esta mayor conciencia sobre las inequidades podría impulsar demandas ciudadanas por políticas sociales y económicas que apunten a lograr mayor solidaridad y cohesión social. Desde una perspectiva sanitaria, el enfoque de salud en todas las políticas, lema de la Unión Europea bajo la presidencia de Finlandia en $2006^{22}$ y que posteriormente fuera adoptado por $\mathrm{OMS}^{23}$, puede ser una buena orientación para actuar sobre los determinantes sociales y fortalecer una mayor equidad, sin olvidar la necesidad de respuesta urgente que requieren grupos especialmente vulnerables como adultos mayores, trabajadores informales o de empleos precarios, con especial atención a mujeres y jóvenes, así como población migrante.

Lo anterior se enlaza con otra dimensión que también se ha relevado durante la pandemia: la salud como derecho humano. Esto cobra especial importancia en un país como Chile, que en estos días se apresta a desarrollar un plebiscito para que la población decida si opta por elaborar una nueva constitución que reemplace a la actual, originalmente elaborada en 1980 durante la dictadura militar. En dicho proceso, el reconocimiento de la salud como derecho es uno de los puntos que está en el centro del debate.

La pandemia de COVID-19 ha hecho evidente para toda la sociedad la necesidad de fortalecer la salud pública, entendida esta como la salud de la población, después que en las décadas de los años 1980 y 1990 las reformas de los sistemas de salud en muchos países de América Latina se centraran en cambios estructurales, financieros y organizacionales, descuidando la salud pública como responsabilidad social e institucional del Estado $^{24}$. Esta mayor valoración de lo público en salud como una instancia absolutamente fundamental para garantizar el derecho a la salud en línea con la resolución de cobertura universal de salud adoptada por la Asamblea Mundial de la Salud de OMS en abril de $2019^{25}$, debe traducirse, entre otros, en recursos humanos que sean apropiados en términos de cantidad y calidad, así como en estrategias para fortalecer los sistemas de vigilancia sanitaria. Un desafío de especial importancia es mejorar sustantivamente los sistemas de información, especialmente respecto de la integración de la información de salud pública y de los sistemas prestadores de atención, de manera que se puedan tomar decisiones oportunas basadas en información confiable.

\section{REFERENCIAS}

1. Jordi Frades (2018). Serie de televisión "La Catedral del Mar" producida por Atresmedia, Televisió de Catalunya y Netflix. Capítulo 5, escena entre minutos 9.01 a 9.23 .

2. Organización Mundial de la Salud (OMS). Peste: Datos y cifras. Temas de Salud. Organización Mundial de la Salud. 31 de octubre de 2017. [Consultado 9 de septiembre 2020].

3. Fuente MJ. Mensajera de la muerte: la peste negra. La aventura de la Historia. 2008;11; 121: 94-8.

4. Sharon N, DeWitte SN. Setting the stage for medieval plague: Pre-black death trends in survival and mortality. Am J Phys Anthr. 2015; 158(3): 441-51.

5. Noymer A, Garenne M. The 1918 Influenza Epidemic's Effects on Sex Differentials in Mortality in the United States. Popul Dev Rev. 2000; 26(3): 565-81.

6. Mizumoto K, Kagaya K, Chowell G. Transmissibility of 2019 Novel Coronavirus: zoonotic vs. human 


\section{COVID-19 EN AMÉRICA LATINA: MÁS ALLÁ DE LOS DATOS EPIDEMIOLÓGICOS ÓSCAR ARTEAGA HERRERA; ALEJANDRA FUENTES GARCÍA}

to human transmission, China, 2019-2020. medRxiv 2020.03.16.20037036.

7. World Health Organization (WHO). Weekly Operational Update on COVID-19. 20 de septiembre de 2020. [Consultado 20 septiembre 2020].

8. World Bank. Projected poverty impacts of COVID19 (coronavirus). The World Bank. Brief. 8 de junio de 2020. [Consultado 15 septiembre 2020].

9. United Nations. The Impact of COVID-19 on Latin America and the Caribbean: Policy Brief. Julio de 2020. [Consultado 15 septiembre 2020].

10. Rosenberg C. (1989) What is an epidemic? AIDS in historical perspective. Daedalus. 1989; 118 (2):1-17.

11. Organización Panamericana de la Salud (OPS/ OMS). Actualización Epidemiológica Enfermedad por coronavirus (COVID-19). 18 de septiembre de 2020. [Consultado 19 septiembre 2020].

12. Johns Hospkins University. Coronavirus Resource Center. 2020. [Consultado 28 septiembre 2020].

13. Mallapaty, S. The coronavirus is most deadly if you are old and male. Nature. 2020; 585: 16-7.

14. Tarlov A. Social determinants of health: the sociobiological translation. En: Blane D, Brunne E, Wilkinson $\mathrm{R}$, editores. Health and social organization: towards a health policy for the twenty-first century. London: Routledge; 1996. p. 71-93.

15. Arroyo C, Cortés T, Engel E, Pardow, D, Simonetti P. Informe sobre la evolución de la epidemia de COVID19 en Chile: Centro de Estudios Espacio Público. 23 de julio de 2020. [Consultado 5 agosto de 2020].

16. Vásquez ML, Vargas I, Unger JP, Mogollón A, Silva MRF, Paepe $P$ de. Integratedhealth care networks in Latin America: toward a conceptual framework for analysis. Rev Panam Salud Publica. 2009;26(4):360-367.
17. Organización Panamericana de la Salud (OMS). Redes integradas de servicios de salud. Conceptos, opciones de política y hoja de ruta para su implementación en las Américas. Washington, DC: OPS/OMS, 2010.

18. Encuesta CEP. Estudio Nacional de Opinión Pública $N^{\circ}$ 84. Diciembre 2019. [Consultado 29 septiembre 2020].

19. Latinobarómetro. Opinión Publica Latinoamericana. Informe 2018. [Consultado 29 septiembre 2020].

20. Frisancho $V$, Vera-Cossio D. Brechas de género en tiempos de la COVID-19. Banco Interamericano de Desarrollo (BID). 1 de julio de 2020 [Consultado 21 septiembre 2020].

21. Bott S, Guedes A, Goodwin, M, Adams Mendoza J. Violencia contra las mujeres en América Latina y el Caribe: Análisis comparativo de datos poblacionales de 12 países. Organización Panamericana de la Salud. Washington, DC : OPS, 2014.

22. European Observatory on Health Systems and Policies. Health in All Policies: Prospects and potentials. En: Ståhl T, Wismar M, Ollila E, Lahtinen E, Leppo, K, editores. Helsinki, Finlandia: Ministry of Social Affairs and Health. 2006.

23. World Health Organization (WHO). Health in all policies: Helsinki statement. Framework for country action. The 8th Global Conference on Health Promotion. WHO Library Cataloguing in Publication; Finlandia. 2014.

24. Muñoz F, López-Acuña D, Halverson P, Guerra de Macedo C, Hanna W, Larrieu M. et al. Las funciones esenciales de la salud pública: un tema emergente en las reformas del sector de la salud. Rev Panam Salud Publica 2000;8(1/2): 126-34.

25. World Health Organization (WHO). World Health Assembly Update. 22 de mayo de 2019. [Consultado 29 septiembre 2020$]$. 


\section{COVID-19 EN AMÉRICA LATINA: MÁS ALLÁ DE LOS DATOS EPIDEMIOLÓGICOS \\ ÓSCAR ARTEAGA HERRERA; ALEJANDRA FUENTES GARCÍA}

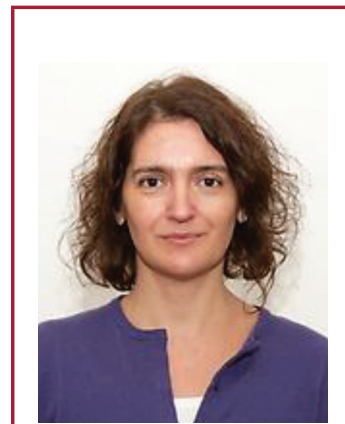

Alejandra Fuentes García. Socióloga (Universidad de Chile), Master en Public Health (Sheffield Hallam University) y Doctora en Salud Pública (U. de Chile). Diplomada Antropología Médica. Es profesora de pre y postgrado en la Escuela de Salud Pública de la Facultad de Medicina de la Universidad de Chile. Fue Directora Académica del Magister en Salud Pública de la Universidad de Chile (2014-2018). Ha sido investigadora en varios proyectos. Actualmente integra el equipo del proyecto ICOVID Chile, iniciativa que agrupa a tres universidades lideradas por la Universidad de Chile, cuyo propósito es el monitoreo de la pandemia de COVID-19 para entregar información oportuna a autoridades, ciudadanía y comunidad científica para guiar la toma de decisiones.

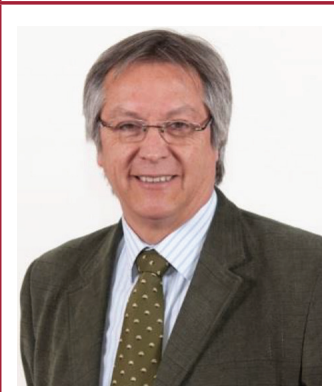

Óscar Arteaga Herrera. Médico y Magíster en Administración en Salud de la Universidad de Chile; MSc Health Services Management y Doctor in Public Health de la Universidad de Londres (London School of Hygiene and Tropical Medicine). Es profesor de pre y postgrado en la Escuela de Salud Pública de la Facultad de Medicina de la Universidad de Chile. Ha sido consultor en sistemas y servicios de salud de OPS/OMS y otros organismos internacionales (Banco Interamericano de Desarrollo, Banco Mundial, OEA y Naciones Unidas). Ha sido investigador en proyectos nacionales e internacionales. Fue Director de la Escuela de Salud Pública de la Universidad de Chile (2011-2017). 